\title{
Percepção e Processamento Visual da Forma: Discutindo Modelos Teóricos Atuais
}

\author{
Natanael Antonio dos Santos \\ Maria Lúcia de Bustamante Simas ${ }^{1} 2$ \\ Universidade Federal de Pernambuco
}

\begin{abstract}
Resumo
Este trabalho discute alguns aspectos teóricos sobre os modelos detector de características e análise de freqüência espacial em cinco seções. A primeira mostra uma breve história e a idéia básica de cada modelo. A segunda mostra evidências psicofísicas e neurofisiológicas sobre a seletividade à freqüência espacial. A terceira, a quarta e a quinta mostram aspectos controversos sobre estes modelos: freqüência espacial versus detectores de características, filtragem local versus global e processamento espacial linear versus não-linear, respectivamente. Um dos objetivos deste trabalho foi discutir tópicos importantes sobre percepção e processamento visual da forma.

Palavras-chave: Visão espacial; percepção visual da forma; processamento visual; detector de características; freqüência espacial.
\end{abstract}

\section{Perception and Visual Processing of Form: Discussing Contemporary Models}

\begin{abstract}
This work discusses some aspects about feature detectors and spatial frequency analysis models in five sections. The first section describes briefly historical aspects and the basic rationale idea of each model. The second section shows the neurophysiological and psychophysical evidences about spatial frequency selectivity. The third, the fourth and the fifth sections show controversial aspects about these models: spatial frequency versus feature detectors, global versus local filtering and nonlinear versus linear spatial processing, respectively. One of the goals of this paper is to discuss important aspects about perception and visual processing of form.

Keywords: Spatial vision; visual perception of forms; spatial processing; feature detectors; spatial frequency.
\end{abstract}

\section{Processamento Visual de Forma: Alguns Modelos}

Em percepção visual da forma, o reconhecimento de cenas visuais ou padrões é um processo fundamental. A idéia da decomposição de objeto complexo dentro de subunidades elementares e o fato de que cada célula individual da via retina-genículo-estriado (via de condução que vai da retina ao córtex visual estriado passando pelo núcleo geniculado lateral) poder responder apenas a certos atributos da imagem levaram vários pesquisadores a investigarem estímulos visuais elementares e/ou primitivos com o objetivo de tentar identificar os atributos ou as subunidades nas quais um padrão complexo é decomposto e processado. Nesse sentido, para investigar como o sistema visual humano, SVH, processa as características espaciais de padrões visuais, surgiram alguns

\footnotetext{
${ }^{1}$ Endereço para correspondência: Laboratório de Percepção Visual, Departamento de Psicologia, $9^{\circ}$ andar, Centro de Filosofia e Ciências Humanas, UFPE, 50670-901, Recife, PE. Fone: (81) 32713734, Fax: (81) 32718271.Email: natanael@npd.ufpe.br

${ }^{2}$ Autores bolsistas CNPq (Processos DCR/CNPq 300746/99-7 e CNPq 523792/95-0, respectivamente).
}

modelos teóricos que podem ser classificados como: modelo de detectores de características, modelo de canal simples e modelo de canais múltiplos.

O modelo de detectores de características defende linhas, bordas e quinas como estímulos primitivos e naturais que compõem os objetos, ao passo que o modelo de canal simples e o de canais múltiplos defendem a propriedade de linearidade como uma característica essencial do sistema. Logo, os modelos de canal simples e canais múltiplos estão entre as tradições que destacam a análise de sistema linear.

\section{Modelo de Detectores de Características}

Este surgiu em paralelo aos modelos de análises de sistemas lineares e foi o modelo dominante durante as décadas de 60 e 80 . O modelo de detectores de características procurou demonstrar a existência de mecanismo detector de características (linhas, bordas e quinas denominados de estímulos primitivos) através do qual o "cérebro" decompõe a imagem física em uma grande quantidade de canais anatômicos que serão 
conectados e formarão posteriormente representações corticais de padrões mais e mais complexos nas áreas superiores.

O modelo de detectores de características teve sua origem no estudo clássico de campo receptivo de células do córtex estriado de gato e macaco (Hubel \& Wiesel, 1962, 1968). Os achados de Hubel e Wiesel mostraram que as células da área 17 do gato respondem preferencialmente ou exclusivamente para estímulos espaciais com certas estruturas. Em outras palavras, estas células tinham preferência para orientação espacial e pareciam responder melhor a linha clara ou escura de largura específica, assim como a bordas de larguras específicas em diversas orientações espaciais. Campo receptivo da célula é a área da retina que quando estimulada provoca uma resposta da célula que pode ser observada através de registo unitário.

O modelo de detectores de características também é referenciado na literatura como modelo hierárquico ou serial, visto que Hubel e Wiesel (1962) propuseram um modelo de conexão com ordem crescente de especificidade dos campos receptivos, de forma que as células do núcleo geniculado lateral passariam informações às células simples, destas para as células complexas e destas para as células hipercomplexas dos tipos I e II, no córtex visual.

Os achados de Hubel e Wiesel $(1962,1968)$ foram valiosos para os pesquisadores interessados em processamento visual de forma. A existência no córtex de mamíferos de células que pareciam extrair informações de tais características, e até mesmo de características mais complexas, foi um grande impulso na área de percepção visual da forma.

Alguns trabalhos psicofísicos também argumentam na direção de detectores de bordas e quinas (King-Smith \& Kulikowski, 1975; Kulikowski \& King-Smith, 1973; Shapley \& Tolhurst, 1973). Eles mediram a sensibilidade para barras e bordas superpostas sobre barras ou grades senoidais de contraste com níveis sub-limiares e interpretaram seus resultados como evidências para classes de detectores que respondem a barras e bordas, e contra a decomposição de freqüência espacial ou o modelo de canais múltiplos.

\section{Modelo de Canal Simples}

A aplicação de grade senoidal para analisar as propriedades espaciais do SVH começou com Schade (1948) e Selwyn (1948). Grade senoidal é um estímulo elementar cuja luminância varia senoidalmente no espaço, em uma direção, em um sistema de coordenadas cartesianas.
O termo "canal simples" está relacionado ao fato de que os métodos iniciais, que aplicavam a análise de Fourier para investigar o processamento visual, consideravam o sistema como filtro único (DePalma \& Lowry, 1962; Lowry \& DePalma, 1961; Schade, 1948, 1956; Selwyn, 1948). De acordo com Braddick, Campbell e Atkinson (1978), o modelo de canal simples considera o sistema como um filtro espacial cujas características são definidas pela função de transferência de modulação, onde o sinal de saída deste sistema é a versão filtrada do sinal de entrada, por exemplo, uma onda espacial senoidal. $\mathrm{Na}$ verdade, a abordagem de canal simples foi uma boa metodologia para obtenção de um bom resumo matemático da maneira pela qual o sistema visual recebe e atenua a informação (Simas, 1985). Em outras palavras, este modelo se restringe a produzir descrições matemáticas das características do sistema visual em resposta ao contraste de diferentes freqüências espaciais.

A importância desse modelo reside no fato de que as respostas para estímulos visuais complexos podem ser preditas a partir de respostas para estímulos com freqüências espaciais elementares através do uso da série de Fourier. O poder preditivo talvez tenha sido a principal razão para se propor tal modelo (Campbell, Carpenter \& Levinson, 1969a).

Freqüência espacial é o número de ciclos (ou períodos ou listras claras e escuras) por medida de espaço, que em percepção visual da forma foi convencionalmente denominado de ciclo por grau de ângulo visual (cpg). Por exemplo, uma freqüência espacial de $1 \mathrm{cpg}$, teria uma listra clara e uma escura por grau de ângulo visual.

\section{Modelo de Canais Múltiplos}

Em 1968, Campbell e Robson sugeriram que o limiar para grade quadrada seria predito a partir da estimativa para grades senoidais. Ou seja, Campbell e Robson observaram que um padrão complexo (onda quadrada ou dente de serra) seria detectado quando o contraste de qualquer um dos seus harmônicos atingisse o limiar se apresentado sozinho. O limiar para uma onda quadrada ou dente de serra de alta freqüência seria o limiar do componente fundamental. Entretanto, para grades de freqüências espaciais baixas, por exemplo, abaixo de um ciclo por grau de ângulo visual, cpg, a sensibilidade ao contraste declina rapidamente, enquanto que a sensibilidade para grades quadradas permanece em níveis relativamente altos. Campbell e Robson argumentaram que a sensibilidade para o terceiro harmônico sozinho presente em tal grade seria suficiente para explicar o aumento proporcional observado. A partir de seus achados, Campbell e Robson sugeriram a existência de 
canais de freqüências espaciais múltiplos. De acordo com este modelo, o sistema visual decompõe o estímulo complexo em seus componentes elementares (ondas senoidais ou cossenoidais), um tipo de análise de Fourier.

Análise de Fourier é a decomposição de qualquer forma de onda complexa ou arbitrária em ondas senoidais e/ou cossenoidais. Enquanto o processo inverso, síntese é a composição de qualquer estímulo complexo pela soma de ondas senoidais e/ou cossenoidais. Neste sentido, uma onda quadrada, por exemplo, de freqüência fundamental $f$, é obtida pela soma de todos os harmônicos ímpares inteiros de $f$. Isto é, $\mathrm{f}+3 \mathrm{f}+5 \mathrm{f}+7 \mathrm{f}+\ldots+\mathrm{nf}$, onde $f$ seria a freqüência fundamental e as outras freqüências seriam os harmônicos ímpares da fundamental.

\section{Seletividade à Freqüência Espacial}

O modelo de canais múltiplos assume que cada faixa estreita de freqüência espacial é conduzida por um canal independente e a função de sensibilidade ao contraste (Contrast Sensitivity Function ou CSF) ou a função de transferência de modulação (Modulation Transfer Function ou MTF) é o envelope da sensibilidade para a série total de canais separados, cada um sensível a uma região restrita do espectro (Braddick e colaboradores, 1978; Graham \& Nachmias, 1971). Em 1974, Sekuler afirmou que se cada um desses canais fosse estimado independentemente teríamos de forma genérica um modelo de canais múltiplos no processamento visual de forma.

Do ponto de vista comportamental ou psicofísico, um canal seria, por exemplo, um filtro com estrutura independente no sistema, que lida com uma faixa estreita de freqüência espacial. Já do ponto de vista fisiológico, um canal significa uma população de neurônios sintonizados para uma faixa estreita de freqüência espacial. Consequentemente, as técnicas fisiológicas podem caracterizar a resposta de um elemento particular do sistema visual, enquanto os paradigmas psicofísicos lidam necessariamente com a resposta do sistema como um todo.

\section{Evidências Psicofísicas para Canais Múltiplos de Freqüência Espacial}

Gilinski (1968) relatou que depois de observar um estímulo listrado de contraste alto por um curto período de tempo, os voluntários podem mostrar um prejuízo para perceber padrões de freqüência e orientação idênticas, mas de contraste mais baixo. Esse fenômeno descrito por Gilinski se tornou conhecido como "adaptação" e foi observado ser altamente específico tanto para freqüência espacial como para orientação (Blakemore \& Campbell, 1969a; Blakemore \& Campbell, 1969b;
Braddick e colaboradores, 1978; De Valois \& De Valois, 1980, 1988; Menees, 1998; Sekuler, 1974; Wilson, Levi, Maffei, Rovamo \& De Valois, 1990). Neste sentido, o fenômeno de adaptação foi introduzido na literatura de percepção e processamento visual de forma como uma técnica capaz de estimar a largura de banda de filtros de freqüências espaciais. $\mathrm{O}$ uso desta técnica como tal se baseia na aceitação de que a diminuição observada na sensibilidade de contraste é proporcional ao grau de excitação do mecanismo detector, cujo pico de sensibilidade à freqüência espacial coincide com a freqüência da grade de teste. Em outras palavras, se as freqüências espaciais são suficientemente diferentes, elas são transmitidas através de canais específicos e independentes e se uma freqüência espacial afeta a detecção de outra freqüência espacial semelhante, é porque elas estão interagindo de alguma forma no mesmo canal. $\mathrm{O}$ fenômeno de adaptação seletiva tanto para freqüência espacial como para orientação espacial fornece apoio ao modelo de filtro para configurações espaciais ou mecanismos seletivos a freqüências espaciais.

Uns dos primeiros e mais convincentes estudos favoráveis à existência de canais múltiplos sensíveis a faixa limitada de freqüência espacial foi realizado por Blakemore e Campbell (1969b). Com a técnica de adaptação seletiva, Blakemore e Campbell verificaram que a adaptação a grade senoidal eleva o limiar da grade senoidal próxima a freqüência adaptada. No mesmo trabalho, eles também investigaram o efeito do terceiro harmônico (3f) e encontraram evidências que deram suporte à hipótese de filtragem de freqüência espacial. Isto é, Blakemore e Campbell observaram que depois da adaptação a uma grade quadrada, uma perda de sensibilidade ao contraste ocorre não só para o fundamental $f$ de tal grade quadrada, mas também para seu harmônico $3 f$. Com base em seus resultados, eles estimaram as larguras de bandas dos canais como sendo da ordem de uma oitava e relataram que o processo de adaptação ocorre a nível cortical devido à comunicação interocular.

Os achados de Blakemore e Campbell (1969b) foram reforçados por Tolhurst (1972) que forneceu mais evidências sobre a elevação do limiar no fundamental e no terceiro harmônico.

Existem outros estudos que fornecem evidências para canais múltiplos e sensibilidade para freqüência espacial usando o paradigma da adaptação seletiva (Blakemore, Nachmias \& Sutton, 1970; Bodinger, 1978; De Valois \& De Valois, 1988; Graham, 1972; Jones \& Tulunay-Keesey, 1975; Menees, 1998; Plante \& Sekuler, 1968; Stromeyer, Klein, Dawson \& Spillmann, 1982; Sullivan, Georgeson 
\& Oatley, 1972; Wilson e colaboradores, 1990), mascaramento de banda crítica (Abadi \& Kulikowski, 1973; Bowen \& Wilson, 1994; Dakin \& Hess, 1997; De Valois \& De Valois, 1988; Levinson \& Sekuler, 1975; Olds \& Engel, 1998; Polat \& Sagi, 1993; Stromeyer e colaboradores, 1982; Wilson e colaboradores, 1990; Wilson, Nagy, Mets \& Perrera, 1988), detecção de estímulo (Graham \& Nachmias, 1971), métodos dos limites (Ellemberg, Lewis, Liu \& Maurer, 1999) e métodos dos estímulos constantes (Peterzell \& Teller, 1996).

\section{Comentário}

Dentre as correntes de modelos de canais múltiplos, Wilson e colaboradores propuseram um modelo com quatro filtros, o qual foi estendido mais tarde para abranger seis filtros (Wilson \& Gelb, 1994; Wilson, 1983; Wilson, McFarlane \& Phillips, 1983). Dois desses, tendo pico de sensibilidade por volta de 0,75 e 1,5 cpg, cobrem freqüências baixas até cerca de $4 \mathrm{cpg}$. Os outros quatro filtros, tendo pico de sensibilidade em 2,8; 4,4; 8,0; e 16 cpg, cobrem freqüências altas, até 32 cpg. Peterzell e Teller (1996), estudando recém-nascidos, obtiveram dados consistentes com a hipótese de dois canais com pico de sensibilidade abaixo de $1 \mathrm{cpg}$. Existem outras correntes de modelos de canais múltiplos (Campbell, Nachmias \& Jukes, 1970; Hirsch \& Hylton, 1982; Humanski \& Wilson, 1993; Regan, Bartol, Murray \& Beverley, 1982; Watson \& Robson, 1981; Yager \& Kramer, 1991). Alguns desses modelos foram discutidos brevemente por Regan e Beverley (1983).

\section{Evidências Fisiológicas para Canais Múltiplos de Freqüência Espacial}

Em 1953, Kuffler estudou padrões de descargas e a organização funcional da retina de mamíferos e registrou disparos para células ganglionares do tipo on-off ou off-on . Posteriormente, Hubel e Wiesel (1962, 1968) demonstraram em gato e macaco, que as células ganglionares da retina e do núcleo geniculado lateral, NGL, e do córtex estriado têm campos receptivos com regiões que são excitatórias (on) e inibitórias (off). Nas células da retina e do NGL a configuração desses campos receptivos eram circulares com centro negativo ou positivo em oposição à periferia. Nas células do córtex estriado esta configuração era similar, porém alongada.

Hubel e Wiesel $(1959,1962,1965,1968)$ demostraram que as células das áreas 17 e 18 do gato e macaco têm uma organização espacial altamente ordenada no que concerne a preferência por orientação espacial. Quando a penetração de microeletrodos era perpendicular à superfície do córtex, a preferência celular por orientação espacial era praticamente a mesma. A porção cortical na qual as células têm a mesma preferência por orientação espacial foi denominada de "coluna de orientação espacial". Posteriormente, foi demonstrado que a seletividade variava de célula para célula em macaco (De Valois, Yund \& Hepler, 1982b; Schiller, Finlay \& Volman, 1976).

Atualmente, as áreas visuais de Brodmann 17, 18 e 19 estão sendo funcionalmente referendadas, como áreas visuais V1, V2-V3 e V4-V5(ou MT) respectivamente, principalmente no macaco (Gallant, Connor, David \& Van Essen, 1998; Kobatake \& Tanaka; 1994; Movshon \& Newsome, 1996). Entretanto, no caso do sistema visual de gato e outros mamíferos ainda continua se utilizando extensamente a classificação de Brodmann (Morley \& Vickery, 1997; Murphy \& Sillito, 1996; Sengpiel, Baddeley, Freeman, Harrad \& Blakemore, 1998; Thompson, Baker, Smyth, Krug \& Tolhurst, 1997a; Thompson, Krug \& Tolhurst, 1997b).

Logo após os estudos psicofísicos (Blakemore \& Campbell, 1969b; Campbell \& Robson, 1968; Sachs, Nachmias \& Robson, 1971), introduzindo a idéia de canais múltiplos seletivos à freqüência espacial, e aos estudos eletrofisiológicos de Hubel e Wiesel (1959, 1962, 1965, 1968), mostrando preferência celular para orientação espacial, notou-se um esforço concentrado na procura de células na retina, no NGL e no córtex estriado que dessem suporte inicial aos estudos comportamentais. Neste sentido, surgiram os estudos neurofisiológicos evidenciando que partes das células corticais são razoavelmente e estreitamente sintonizadas para freqüências espaciais (Campbell, Cooper \& EnrothCugell, 1969b; Campbell, Cooper, Robson, \& Sachs 1969c; Campbell \& Maffei, 1974; De Valois, Albrecht \& Thorell, 1982a; De Valois \& De Valois, 1988; Geisler \& Albrecht, 1997; Ikeda \& Wrigth, 1975; Maffei \& Fiorentini, 1973; Movshon, Thompson \& Tolhurst, 1978; Schiller e colaboradores, 1976; Thompson e colaboradores, 1997a, 1997b; Tootell, Silverman \& De Valois, 1981; Wilson e colaboradores, 1990). Os achados mais notáveis foram aqueles que mostraram que as células corticais simples eram mais estreitamente sintonizadas para freqüências espaciais do que as células ganglionares da retina e do NGL, e que a maioria das células simples foi sensivelmente sintonizada para bandas de freqüências espaciais bem definidas e, praticamente insensível, para freqüências espaciais uma oitava acima ou abaixo do seu pico de sintonia (Maffei, Fiorentini \& Bisti, 1973). Esta sintonia estreita das células simples para freqüência espacial foi o substrato neural para os canais de freqüência espacial postulados com base nos experimentos psicofísicos 
(Blakemore \& Campbell, 1969a; Campbell \& Robson, 1968; Sachs e colaboradores, 1971). Oitava é uma alteração na freqüência por um fator de dois. Em outras palavras, oitavas de freqüência espacial são incrementos iguais em uma escala logarítmica (Blakemore e colaboradores, 1970).

Outros estudos merecem destaque particular por mostrarem a existência de organização arquitetural no córtex visual. Por exemplo, Maffei e Fiorentini (1977) estudaram a distribuição de células corticais de gato. Os resultados foram muito sugestivos e mostraram preferência à freqüência espacial na área 17. A arquitetura de células corticais, considerando a preferência por freqüência espacial do estímulo, parecia complementar a orientação espacial do estímulo e vice-versa. Ou seja, em penetrações perpendiculares à superfície do córtex, as células mostraram a mesma preferência por orientação espacial, ao mesmo tempo em que tinham preferência variada por freqüência espacial. Já em penetrações paralelas à superfície do córtex, observou-se o contrário, a preferência por orientação espacial muda de célula para célula, mas a preferência por freqüência espacial continua praticamente invariante. Assim, vieram os primeiros indícios que as células sintonizadas para diferentes faixas de freqüências espaciais podiam estar organizadas em camadas diferentes do córtex como a organização encontrada para orientação (Hubel \& Wiesel, 1974). Mais tarde, Silverman (1984) e Tootell e colaboradores (1981), usando a técnica de 2-desoxi-D-glicose (2-DG) no córtex de gato, mostram evidências para organização colunar de freqüências espaciais semelhante àquela encontrada para orientação por Hubel e Wiesel (1974). No trabalho de 1988, De Valois e De Valois apresentaram modelos esquemáticos da organização colunar do gato e macaco. De acordo com os modelos propostos por De Valois e De Valois, em cada região de dominância ocular são arranjadas colunas de células sintonizadas para freqüências espaciais e orientações diferentes, de tal forma que a combinação freqüência espacial/orientação ocorre em cada um módulo cortical.

\section{Comentários}

1) Thompson e colaboradores (1997a, 1997b) demostraram que o furão (ferret), um animal de laboratório, também apresenta sintonia para freqüência espacial. $\mathrm{Na}$ verdade, Thompson e colaboradores comparam as respostas de neurônios das áreas 17 e 18 do furão com as mesmas áreas do gato. Os resultados mostraram que as respostas dessas duas áreas do furão para grade senoidal são semelhantes às respostas das mesmas áreas no gato. Isto é, as áreas 17 e 18 do furão demonstraram sintonia para freqüência espacial.

2) As evidências fisiológicas mostram que na região da fóvea em macaco o pico de várias células se encontra sobre uma faixa de uma oitava e que todas as células juntas cobrem aproximadamente quatro oitavas (Campbell e colaboradores, 1969c; De Valois, Albrecht \& Thorell, 1977; De Valois \& De Valois, 1988). Resultados similares são encontrados para o gato se as áreas 17 e 18 são consideradas juntas (Movshon e colaboradores, 1978). Neste sentido, parece que o sistema visual de cada uma dessas espécies (gato, macaco e humano) cobre uma faixa de aproximadamente 4 oitavas de freqüências espaciais, sendo que a posição da faixa varia no espectro da freqüência.

3) Outro tipo de evidência é que algumas células na área próxima a fóvea são sintonizadas para freqüências espaciais baixas, da mesma forma que outras são sintonizadas para freqüências altas (Carrasco, Mclean, Katz \& Frieder, 1998; Graham, Robson \& Nachmias, 1978). Esses achados, mais uma vez, são consistentes com as evidências psicofísicas discutidas acima mostrando canais múltiplos dentro do sistema que processa informação da fóvea e da periferia. Entretanto, a sensibilidade ao contraste declina através do campo visual, isto é, dependendo da propriedade espacial do estímulo, a sensibilidade ao contraste declina mais rapidamente como uma função da excentricidade para freqüências espaciais mais altas do que para freqüências espaciais mais baixas (Hess \& Hayes, 1994).

\section{Filtro de Freqüência Espacial versus Detectores de Características}

Os resultados comportamentais e eletrofisiológicos revisados na seção anterior sugerem que o sistema visual possui os atributos necessários para uma análise de freqüência espacial da informação visual. De certo modo, apoiando esta conclusão, estão neurônios sensíveis a amplitude, fase, e freqüência espacial. Estes neurônios são sensíveis ao fenômeno de adaptação com grades senoidais, de maneira tal que parecem diretamente correlacionados com a psicofísica (Maffei \& Fiorentini, 1973; Maffei e colaboradores, 1973; Pollen, Lee \& Taylor, 1971). Em 1972, Maffei e Fiorentini realizaram um experimento psicofísico interessante. Eles pegaram duas grades senoidais, o primeiro e o terço harmônico de uma onda quadrada, e apresentaram separadamente aos dois olhos de um voluntário humano. O que o voluntário percebeu foi um padrão composto, o resultado da superposição neural das duas grades. O padrão percebido foi uma onda quadrada bem parecida com o padrão 
visto monocularmente quando as duas grades eram sobrepostas fisicamente. Concluiu-se com esse experimento que a informação sobre amplitude e fase dos dois estímulos senoidais apresentados separadamente aos dois olhos pode ser sintetizada pelo sistema visual.

Existem outros resultados mostrando que, na área 18 do córtex do gato, algumas células complexas respondem melhor para grades do que para qualquer outro estímulo visual (Glezer, Ivanoff \& Tscherbach, 1973; Pollen \& Ronner, 1975).

Em resumo, o peso das evidências psicofísicas, fisiológicas e anatômicas em favor de freqüência espacial não exclui a possibilidade de que filtros de freqüências espaciais possam coexistir em harmonia com certas famílias de detectores especializados de características. Por exemplo, Maffei (1978) argumentou que é razoável e talvez econômico para o sistema nervoso desenvolver um sistema especializado para aquelas características pertinentes à vida do animal. Entretanto, até o momento, não se definiram quais seriam essas características pertinentes e nem como estudar a infinidade de características que podem existir. $\mathrm{Na}$ verdade, os estudos nesta direção ainda são escassos e difíceis de serem replicados. Ainda assim, mesmo existindo poucas evidências na direção de detectores de características no sistema visual e uma grande quantidade de evidências a favor de análise de freqüência espacial, esta última não inviabiliza a existência de detectores de características. $\mathrm{O}$ modelo de Marr (1976), por exemplo, postula filtragem de freqüência espacial como um estágio preliminar de um sistema detector de características como por exemplo, barras. Só que, se existem detectores de características, eles possivelmente estariam além da área $17 \mathrm{ou}$ V1, depois de um estágio de filtragem de freqüência espacial, pois a detecção de bordas é um problema complicado e envolve possivelmente tipos de células diferentes das encontradas no córtex estriado (De Valois \& De Valois, 1980, 1988). Por outro lado, Georgeson e Meese (1997) mencionam que a análise de Fourier local e a análise de característica parecem ser estágios sucessivos no processamento visual de forma.

\section{Filtragem Espacial Linear Local versus Global}

Atualmente, na análise de sistema linear, há divergência quanto à filtragem de freqüência espacial, pois existem duas abordagens com propósito um tanto diferente. Filtros de freqüências espaciais podem ocorrer sob o ponto de vista local ou parte por parte ou patch-by-patch (De Valois \& De Valois, 1988; Gray \& Regan, 1998; Robson, 1975); ou sob o ponto de vista global, isto é filtragem de uma faixa grande do campo visual (Pollen e colaboradores, 1971; Pollen \& Ronner, 1975). A filtragem local considera a análise sob a extensão das dimensões convencionais do campo receptivo, o que corresponde a algo entre alguns minutos de arco a um ou dois graus dependendo da sintonia espacial da célula. A filtragem global, por outro lado, considera que as células fazem uma análise de Fourier através de uma faixa estreita de todo campo visual ao invés de uma pequena parte (Pollen e colaboradores, 1971). Vejamos a seguir alguns aspectos da análise local versus global e para mais detalhes ver, por exemplo, De Valois e De Valois (1988).

A análise local tem como base: 1) o espaço visual não é infinito, ele é localizado em nível de campo receptivo; 2) os objetos geralmente são formados por freqüências espaciais altas, por exemplo, textura; 3) a função de Gabor tem se destacado na análise do sistema visual (Daugman, 1984; Geri, Lyon \& Zeevi, 1995; Graham, Sutter \& Venkatesan, 1993; Hess \& Wilcox, 1994; Westheimer, 1998). Um aspecto importante da função de Gabor (filtros de freqüência localizado em envelope Gaussiânico) é como ondas senoidais e cossenoidais puras podem fornecer uma descrição completa e precisa de qualquer forma de onda complexa (Helstrom, 1966).

A análise global tem como base: 1) a análise de Fourier é em princípio um processo global. Ou seja, a amplitude de Fourier para uma determinada freqüência espacial e orientação é determinada pela forma de onda naquela orientação estendida infinitamente no espaço; 2) a análise de Fourier pode ser aplicada em qualquer situação real, pois mesmo o espaço visual não sendo infinito, o campo visual segundo De Valois e De Valois (1988) pode se estender a 200 graus ou mais; 3) a análise global é essencial para estímulos visuais com alta periodicidade, como por exemplo, grades senoidais, freqüências radiais, freqüências angulares, etc.; 4) a abordagem de Fourier pode fornecer a análise ou a síntese de qualquer estímulo visual complexo levando em consideração a freqüência espacial, a fase e a orientação; 5) dados obtidos com padrões de freqüências radiais, angulares e hipérboles não podem ser explicados por processamento de contorno local (Wilkinson, Wilson \& Habak, 1998; Wilson \& Wilkinson, 1997, 1998). Em síntese, a análise de Fourier é uma ferramenta sólida, clara e tem em seu favor a vantagem de fazer predições quantitativas. Neste sentido, pode especificar combinações de um número finito de componentes elementares dependendo da precisão exigida. Ondas moduladas por seno ou cosseno são elementos básicos ou elementares da análise de Fourier.

\section{Comentário}

O modelo de canais múltiplos mais viável depende, entretanto, principalmente do objeto de estudo e do tipo 
de padrão em questão (periódico, aperiódico ou com pouca periodicidade). No caso de quem tem interesse em estudar e caracterizar a resposta do sistema visual para padrões de freqüências espaciais baixas, médias e altas com alta periodicidade; a análise de Fourier ou o modelo de canais múltiplos focalizando aspectos globais pode ser fundamental, considerando os modelos de canais múltiplos vigentes até o momento.

\section{Processamento Espacial Linear versus Não-Linear}

Em 1966, Enroth-Cugell e Robson descreveram duas classes de células ganglionares na retina, as quais denominaram de células X e células $\mathrm{Y}$. A célula X com campo receptivo pequeno mostrou somação espacial linear e a célula Y com campo receptivo maior não mostrou somação espacial linear (De Valois e colaboradores, 1982a; Enroth-Cugell \& Robson, 1966). Então, é possível que existam duas grandes vias no sistema visual, uma onde a linearidade ou a presença de somação linear em sub-regiões de seus campos receptivos é relevante para o processamento espacial - formada pelas células $\mathrm{X}$ da retina, NGL, células corticais simples, e outra onde a linearidade ou somação não é relevante para o processamento espacial - formada pelas células $\mathrm{Y}$ da retina, do NGL e células corticais complexas (De Valois e colaboradores, 1982a; De Valois \& De Valois 1988; Zhou \& Baker, 1996). A denominação de células X e Y é específica para o gato; no caso de macacos e humanos, o sistema que envolve as células com propriedades semelhantes as células $\mathrm{X}$ e Y recebem as denominações de sistema parvocelular e magnocelular, respectivamente. Sistema linear é aquele cuja resposta é proporcional ao estímulo, ou seja, aumentando-se a intensidade de luminância, aumenta-se a intensidade da imagem na mesma proporção.

\section{Comentário}

A aplicação da análise de sistema linear em visão, à primeira vista, não parece ser apropriada, pois o sistema visual é altamente não linear. Entretanto, a presença de não-linearidade em sistemas biológicos (p.ex., visão, audição, dentre outros) não inviabiliza ou invalida a aplicabilidade de modelo linear em situações restritas, uma vez que a exigência básica da análise de Fourier é atendida quando o sistema em estudo é linear, nem que seja em condições restritas. Do ponto de vista teórico, qualquer sistema pode ser considerado linear em condições restritas bem estabelecidas, pois até mesmo uma função quadrática (exponencial, logarítmica ou cúbica) pode ser considerada linear em um intervalo suficientemente restrito. Na prática, certamente, o processo pode ser bem mais complexo.
Foram relatados acima vários estudos com organismos e métodos diferentes que mostram que os resultados psicofísicos e neurofisiológicos convergem de alguma forma para um dado modelo, especialmente para o modelo de canais múltiplos de freqüência espacial ou filtragem espacial linear. Por outro lado, processamento não-linear é uma denominação utilizada em modelos cognitivos de reconhecimento de objetos, particularmente aqueles que enfatizam o papel do processamento de cima para baixo ou top-down (Olds \& Engel, 1998) ou segregação de textura (De Valois \& De Valois, 1988; Wilson \& Wilkinson, 1997).

Em resumo, os modelos abordados nesse trabalho teoricamente diferem de vários outros modelos ou teorias clássicas em percepção visual de forma, por exemplo, a teoria da Gestalt, que se fundamenta em princípios de organização de figura e fundo tais como: proximidade, fechamento, continuidade, similaridade, simetria, dentre outros. O processamento visual de forma para a Gestalt ocorre de cima para baixo ou top-down, o que é característico da perspetiva cognitiva que estuda a percepção visual da forma com ênfases em esquemas e representações mentais. Ao passo que os modelos discutidos neste trabalho defendem que o processamento visual da forma, que é sintetizada no córtex visual, ocorre graças as informações que chegam e são analisadas na retina, isto é, processamento visual de baixo para cima ou bottom-up (modelos neurofisiológicos e comportamentais).

\section{Consideração Final}

Um modelo, na maioria das vezes, é uma ferramenta matemática que nasce com suas imperfeições, pois quando um modelo vier simular fidedignamente um sistema biológico (p.ex. sistema visual) ele deixar de ser um modelo e passa a ser o próprio sistema. Desta forma é difícil se não impossível produzir um modelo único que solucione todas as indagações, até por que quando se supera uma, surgem dezenas de outras. Logo não existe um modelo perfeito. Qualquer um dos modelos mencionado aqui dentre outros não citados pode ser de grande importância para compreender o processamento espacial realizado pelo sistema visual. O modelo mais viável depende dentre outras coisas (p. ex. estímulos visuais) do objetivo do experimento. Entretanto, o modelo de canais múltiplos ou análise de sistema linear continua sendo um dos mais requisitados para estudar e caracterizar a resposta do sistema visual humano.

Imagine um sistema de camuflagem, uma "casa-mata" camuflando um veículo ou um helicóptero de guerra. 
Um sistema de camuflagem perfeito deveria leva em consideração o processamento bottom-up (modelo de canais múltiplos) e top-down (cognição). Bottom-up porque teria que filtrar as freqüências espaciais baixas, médias e altas. Uma vez que os canais para as freqüências espaciais baixas dão o formato geral do objeto, já os canais para as freqüências espaciais médias dão o formato que se assemelha ao objeto final, enquanto que os canais para as freqüências espaciais altas são responsáveis pelos detalhes finos (preenchimento das quinas ou os contornos). Lembre-se que todo esse processamento se iniciaria na retina onde existe grupo de receptores (cones e bastonetes) com sensibilidade discreta para brilho e contraste. Topdown porque teria que levar em consideração, também, o contexto onde o veículo ou o helicóptero de guerra seria camuflado, pois a experiência do observador poderia denunciar a "casa-mata".

A percepção e processamento visual da forma são processos dinâmicos e contínuos e podem envolver tanto um processamento de baixo para cima ou bottom-up como um processamento de cima para baixo ou top-down. Assim, dependendo da experiência é difícil dizer onde um começa e o outro termina.

Neste contexto, no estudo da percepção visual de forma, existem inúmeros modelos além desses que destacamos neste artigo. Entretanto os três modelos focalizados estão dentro, ou assemelhado, ao âmbito das pesquisas conduzidas em nosso laboratório.

\section{Referências}

Abadi, R.V. \& Kulikowski, J. J. (1973). Linear summation of spatial harmonics in human vision. Vision Research, 13, 1625-1628.

Blakemore, C. \& Campbell, F. C. (1969a). Adaptation to spatial stimuli. Journal of Physiology, 200, 11-12.

Blakemore, C. \& Campbell, F. C. (1969b). On the existence of neurons in the human visual system selectively sensitive to the orientation and size of retinal images. Journal of Physiology, 203, 237-260.

Blakemore, C., Nachmias, J. \& Sutton, P. (1970). The perceived spatial frequency selective neurones in the human brain. Journal of Physiology, $210,727-750$.

Bodinger, D. M. (1978). The decay of grating adaptation. Vision Research, 18, 89-91.

Bowen, R. W. \& Wilson, H. R. (1994). A two-process analysis of pattern masking. Vision Research, 34, 645-657.

Braddick, O., Campbell, F. W. \& Atkinson, J. (1978). Channels in vision: Basic aspects. Em R. Held, H. W. Leibowitz \& H. L. Teuber (Orgs.), Handbook of Sensory Physiology: Perception (Vol. V, pp. 3-38). New York: Springer-Verlag.

Campbell, F. W., Carpenter, R. H. S. \& Levinson, J. Z. (1969a). Visibility of aperiodic patterns compared with that of sinusoidal gratings. Journal of Physiology, 204, 283-298.

Campbell, F. W., Cooper, G. F. \& Enroth-Cugell, C. (1969b). The spatial selectivity of the visual cells of the cat. Journal of Physiology, 203, 223235.
Campbell, F. W., Cooper, G. F., Robson, J. G. \& Sachs, M. B. (1969c). The spatial selectivity of cells of the cat and the squirrel monkey. Journal of Physiology, 204,120-121.

Campbell, F. W. \& Maffei, L. (1974). Contrast and spatial frequency. Scientific American, 231, 106-114.

Campbell, F. W., Nachmias, J. \& Jukes, J. (1970). Spatial-frequency discrimination in human vision. Journal of the Optical Society of America, 60, 555559.

Campbell, F. W. \& Robson, F. G. (1968). Application of the Fourier analysis to the visibility of gratings. Journal of Physiology, 197, 551-566.

Carrasco, M., Mclean, T. L., Katz, S. M. \& Frieder, K. S. (1998). Feature asymmetries in visual search: Effects of display duration, target eccentricity, orientation and spatial frequency. Vision Research, 38(3), 347-374.

Dakin, S. C. \& Hess, R. F. (1997). The spatial mechanisms mediating symmetry perception. Vision Research, 37(20), 2915-2939.

Daugman, J. G. (1984). Spatial visual channels in the Fourier plane. Vision Research, 24(9), 891-910.

De Valois, R. L., Albrecht, D. G. \& Thorell, L. G. (1977). Spatial tunning of LGN and cortical cells in monkey visual system. Em H. Spekreijse \& L. H. van der Tweel (Orgs.), Spatial Contrast (pp. 60-63). Amsterdam: North Holland.

De Valois, R. L., Albrecht, D. G. \& Thorell, L. G. (1982a). Spatial frequency selectivity of cells in macaque visual cortex. Vision Research, 22, 545559.

De Valois, R. L. \& De Valois, K. K. (1980). Spatial vision. Annual Review of Psychology, 31, 309-341.

De Valois, R. L. \& De Valois, K. K. (1988). Spatial vision. New York: Oxford University Press.

De Valois, R. L., Yund, E. W. \& Hepler, N. (1982b). The orientation and direction selectivity of cells in macaque visual cortex. Vision Research, $22,531-544$

DePalma, J. J. \& Lowry, E. M. (1962). Sine-wave response of the visual system. II Sine-wave and square sensitivity. Journal of the Optical Society of America, 52, 328-335.

Ellemberg, D., Lewis, T. L., Liu, C. H. \& Maurer, D. (1999). Development of spatial and temporal vision during childhood. Vision Research, 39, 2325-2333.

Enroth-Cugell, C. \& Robson, J. M. (1966). The contrast sensitivity of retinal ganglion cells of the cat. Journal of Physiology, 187, 512-552.

Gallant, J. L., Connor, C. E. \& Van Essen, D. C. (1998). Neural activity in areas V1, V2, and V4 during free viewing of natural scenes compared to controlled viewing. NeuroReport, 9, 1673-1678.

Geisler, W. S. \& Albrecht, D. G. (1997). Visual cortex neurons in monkeys and cats: Detection, discrimiantion, and identification. Visual Neuroscience, 14, 897-919.

Georgeson, M. A. \& Meese, T. S. (1997). Perception of stationary plaids: The role of spatial filters in edge analysis. Vision Research, 37(23), 3255-3271.

Geri, G. A., Lyon, D. R. \& Zeevi, Y. Y. (1995). Preattentive equivalence of multicomponent Gabor textures in the central and peripheral visual field. Vision Research, 35, 495-506.

Gilinski, A. S. (1968). Orientation-specific effects of patterns of adapting light on visual acuity. Journal of the Optical Society of America, 58, 13-18.

Glezer, V. D., Ivanoff, V. A. \& Tscherbach, T. W. (1973). Investigation of complex and hypercomplex receptive fields of visual cortex of the cat as spatial frequency filters. Vision Research, 13, 1875-1904.

Graham, N. (1972). Spatial frequency channels in the human visual system: Effects of luminance and pattern drift rate. Vision Research, 12, 53-63.

Graham, N. \& Nachmias, J. (1971). Detection of grating patterns containing two spatial frequencies: A comparison of single-channel and multiple channel models. Vision Research, 11, 251-259.

Graham, N., Robson, J. G. \& Nachmias, J. (1978). Grating summation in fovea and periphery. Vision Research, 18, 815-825.

Graham, N., Sutter, A. \& Venkatesan, C. (1993). Spatial-frequency- and orientation-selectivity of simple and complex channels in region segregation. Vision Research, 33, 1893-1911. 
Gray, R. \& Regan, D. (1998). Spatial frequency discrimination and detection characteristics for gratings defined by orientation texture. Vision Research, 38, 2601-2617.

Helstrom, C. W. (1966). An expansion of a signal in Gaussian elementary signals. IEEE Trans. Inf. Theory, IT-13, 81-82.

Hess, R. F. \& Hayes, A. (1994). The coding of spatial position by human visual system: Effects of spatial scale and retinal eccentricity. Vision Research, 34, 625-643.

Hess, R. F. \& Wilcox, L. M. (1994). Linear and nor-linear filtering stereopsis. Vision Research, 34, 2431-2438.

Hirsch, J. \& Hylton, R. (1982). Limits of spatial frequency discrimination as evidence of neural interpolation. Journal of the Optical Society of America, 72, 1367-1374.

Hubel, D. H. \& Wiesel, T. N. (1959). Receptive fields of single neurones in the cat's striate cortex. Journal of Physiology, 148, 574-591.

Hubel, D. H. \& Wiesel, T. N. (1962). Receptive fields, binocular interaction and functional architecture in the cat's visual cortex. Journal of Physiology, 160, 106-154.

Hubel, D. H. \& Wiesel, T. N. (1965). Receptive fields and functional architecture in two nonstriate visual areas (18 and 19) of the cat. Journal of Neurophysiology, 28, 229-289.

Hubel, D. H. \& Wiesel, T. N. (1968). Receptive fields and functional architecture of monkey striate cortex. Journal of Physiology, 195, 215-243.

Hubel, D. H. \& Wiesel, T. N. (1974). Sequence regularity and geometry of orientation columns in the monkey striate cortex. Journal of Physiology, 158, 267-294.

Humanski, R. A. \& Wilson, H. R.(1993). Spatial-frequency adaptation: Evidence for a multiple-channel model of short-wavelength-sensitivecone spatial vision. Vision Research, 5, 665-675.

Ikeda, H. \& Wrigth, M. J. (1975). Spatial and temporal properties of "sustained" and "transient"neurones in area 17 of the cat's visual cortex. Experimental Brain Research, 22, 363-383.

Jones, R. M. \& Tulunay-Keesey, U. (1975). Local retinal adaptation and spatial frequency channels. Vision Research, 15, 1239-1244.

King-Smith, P. E. \& Kulikowski, J. J. (1975). The detection of gratings by independent activation of line detectors. Journal of Physiology, 245, 237-271.

Kobatake, E. \& Tanaka, K. (1994). Neuronal selectivities to complex object features in the ventral visual pathway of the macaque cerebral cortex. Journal of Neurophysiology, 71(3), 856-867.

Kuffler, S. W. (1953). Discharge patterns and functional organiazation of mammalian retina. Journal of Neurophysiology, 16, 37-68.

Kulikowski, J. J. \& King-Smith, P. E. (1973). Spatial arrangement of line, edge and grating detectors relealed by sub-threshold summation. Vision Research, 13, 1455-1478.

Levinson, E. \& Sekuler, R. (1975). The independence of channels in human vison selective for direction of moviment. Journal of Physiology, 250,347-366.

Lowry, E. M. \& DePalma, J. J. (1961). Sine-wave response of the visual system. I. The Mach Phenomenon. Journal of the Optical Society of America, 51, 740-746.

Maffei, L. (1978). Spatial frequency channels: Neural mechanisms. Em R. Held, H. W. Leibowitz, \& H. L. Teuber (Orgs.), Handbook of Sensory Physiology: Perception (Vol. V, pp. 3-38). New York: Springer-Verlag.

Maffei, L. \& Fiorentini, A. (1972). Processes of synthesis in visual perception. Nature, 240, 479-481.

Maffei, L. \& Fiorentini, A. (1973). The visual cortex as a spatial frequency analyzer. Vision Research, 13, 1255-1267.

Maffei, L. \& Fiorentini, A. (1977). Spatial frequency rows in the striate visual cortex. Vision Research, 17, 257-264.

Maffei, L., Fiorentini, A. \& Bisti, S. (1973). Neural correlates of perceptual adaptation to gratings. Science, 182, 1036-1038.

Marr, D. (1976). Early processing of visual information. Philos. Trans. R. Soc. London Ser.(B), 275, 483-524.

Menees, S. M. (1998). The effect of spatial frequency adaptation on the latency of spatial contrast detection. Vision Research, 38, 3933-3942.
Morley, J. W. \& Vickery, R. M. (1997). Spatial and temporal frequency selectivity of cells in area 21a of the cat. Journal of Physiology, 501(2), 405-413.

Movshon, J. A. \& Newsome, W. T. (1996). Visual response properties of striate cortical neurons projecting to area MT in macaque monkeys. The Journal of Neuroscience, 16(23), 7733-7741.

Movshon, J. A., Thompson, I. D. \& Tolhurst, D. J. (1978). Spatial and temporal contrast sensitivity of neurones in areas 17 and 18 of the cat's visual cortex. Journal of Physiology, 283, 101-120.

Murphy, P. C. \& Sillito, A. M. (1996). Connectivity patterns formed by single axons from visual cortical areas 17 and 18 to the lateral geniculate nucleus of the cat. Journal of Physiology, 494, 17.

Olds, E. S. \& Engel, S. A. (1998). Linearity across spatial frequency in object recognition. Vision Research, 38, 2109-2118.

Pantle, A. \& Sekuler, R. (1968). Size detecting mechanisms in human vision. Science, 162, 1146-1148.

Peterzell, D. H. \& Teller, D. Y. (1996). Individual differences in contrast sensitivity functions: The lowest spatial frequency channels. Vision Research, 36, 3077-3085.

Polat, U. \& Sagi, D. (1993). Lateral interactions between spatial channels: Suppression and facilitation revealed by lateral masking experiments. Vision Research, 33, 993-999.

Pollen, D. A., Lee, J. R. \& Taylor, J. H. (1971). How does the striate cortex begin the reconstruction of the visual world? Science, 173, 74-77.

Pollen, D. A. \& Ronner, S. F. (1975). Periodic excitability changes across the receptive fields of complex cells in the striate and parastriate cortex of the cat. Journal of Physiology, 245, 667-697.

Regan, D., Bartol, S., Murray, T. J. \& Beverley, K. I. (1982). Spatial frequency discrimination in normal vision and in patients with multiple sclerosis. Brain, 105, 735-754.

Regan, D. \& Beverley, K. I. (1983). Spatial-frequency discrimination and detection: comparison of postadaptation thresholds. Journal of the Optical Society of America, 73, 1684-1690.

Robson, J. G. (1975). Receptive fields: Neural representation of the spatial and intensive attributes of the visual image. Em E. C. Carterette \& M. P. Fridman (Orgs.), Handbook of Perception (Vol. V, pp. 81-112). New York: Academic Press.

Sachs, M. B., Nachmias, J. \& Robson, J. G. (1971). Spatial frequency channels in human vision. Journal of the Optical Society of America, 61, 1176-1186.

Schade, O. H. (1948). Electro-optical characteristics of television systems. 1. Characteristics of vision and visual systems. RCA Review, 9, 5-37.

Schade, O. H. (1956). Optical and photoelectric analog of the eye. Journal of the Optical Society of America, 46, 721-739.

Schiller, P. H., Finlay, B. L. \& Volman, S. F. (1976). Quantitative studies of single-cell properties in monkey striate cortex. II. Orientation specificity and ocular dominance. Journal of Neurophysiology, 39, 13201333.

Sekuler, R. (1974). Spatial Vision. Annual Review of Psychology, 25, 195-232.

Sengpiel, F., Baddeley, R. J., Freeman, T. C. B., Harrad, R. \& Blakemore, C. (1998). Different mechanisms underlie three inhibitory phenomena in cat area 17. Vision Research, 38, 2067-2080.

Selwyn, E. W. H. (1948). The photographic and visual resolving power of lenses. 1. Visual resolving power. Photographic Journal, 88B, 6-12.

Shapley, R. M. \& Tolhurst, D. J. (1973). Edge detectors in human vision. Journal of Physiology, 229, 165-183.

Silverman, M. S. (1984). Deoxyglucose and electrophysiological evidence for spatial frequency columns in cat striate cortex. Tese de Doutorado não-publicada, University of California, San Francisco, Califórnia, EUA.

Simas, M. L. B. (1985). Linearity and domain invariance in the visual system. Tese de Doutorado não-publicada. Queen's University at Kingston, Ontario, Canada: University Microfilms International. Ann Arbor: Michigan.

Stromeyer, C. F., Klein, S., Dawson, B. M. \& Spillmann, L. (1982). Low spatial-frequency channels in human vision: Adaptation and Masking. Vision Research, 22, 225-233.

Sullivan, G. D., Georgeson, M. A. \& Oatley, K. (1972). Channels for spatial frequency selection and detection of single bars by the human visual system. Vision Research, 12, 383-394. 
Thompson, I. D., Baker, G. E., Smyth, D., Krug, K. \& Tolhurst, D. J. (1997a). Spatial-frequency tuning of neurons in area 17 and area 18 of the anaesthetized ferret. Journal of Physiology, 501, 120.

Thompson, I. D., Krug, K. \& Tolhurst, D. J. (1997b). The spatial frequency tuning of neurons in area 17 of the anaesthetized ferret. Journal of Physiology, 499, 86.

Tolhurst, D. J. (1972). Adaptation to square-wave gratings: Inhibition between spatial frequency channels in the human visual system. Journal of Physiology, 226, 231-248.

Tootell, R. B., Silverman, M. S. \& De Valois, R. L. (1981). Spatial frequency columns in primary visual cortex. Science, 214, 813-815.

Watson, A. B. \& Robson, J. G. (1981). Discrimination at threshold: Labeled detectors in human vision. Vision Research, 21, 1115-1122.

Westheimer, G. (1998). Lines and Gabor functions compared as spatial visual stimuli. Vision Research, 38(4), 487-491.

Wilkinson, F., Wilson, H. R. \& Habak, C. (1998). Detection and recognition of radial frequency patterns. Vision Research, 38, 3555-3568.

Wilson, H. R. (1983). Psychophysical evidence for spatial channels. Heidelberg: Springer-Verlag.

Wilson, H. R. \& Gelb, D. J. (1984). Modified line element theory for spatial frequency and width discrimination. Journal fo the Optical Society of America A, I, 124-131.

Wilson, H. R., Levi, D., Maffei, L., Rovamo, J. \& De Valois, R. (1990). The perception of form: Retina to striate cortex. Em S. W. Spillmann \& J. S. Werner (Orgs.), Visual perception: The Neurophysiological Foundation (pp. 231-271). New York: Academic Press.
Wilson, H. R., McFarlane, D. H. \& Phillips, G. C. (1983). Spatial frequency tuning of orientation selective units estimated by oblique masking. Vision Research, 23, 873-882.

Wilson, H. R., Nagy, S. E., Mets, M. B. \& Perrera, V. P. (1988). Spatial frequency and orientation tunning of spatial visual mechanisms in human albinos. Vision Research, 28, 991-999.

Wilson, H. R., \& Wilkinson, F. (1997). Evolving concepts of spatial channels in vision: From independence to nonlinear interactions. Perception, 26, 939-960.

Wilson, H. R. \& Wilkinson, F. (1998). Detection of global structure in glass patterns: Implications for form vision. Vision Research, 38, 29332947.

Yager, D. \& Kramer, P. (1991). A model for perceived spatial frequency and spatial frequency discrimination. Vision Research, 31, 1067-1072.

Zhou, Y. \& Baker, C. L. J. (1996). Spatial properties of envelope-responsive cells in area 17 and 18 neurons of the cat. Journal of Neurophysiology, 75(3), 1038-1050.

Sobre os autores:

Natanael Antonio dos Santos é Doutor em Neurociências e Comportamento pelo Instituto em Psicologia Experimental da Universidade de São Paulo (1999). Pesquisador do CNPq, Bolsa DCR, Laboratório de Percepção Visual, Departamento de Psicologia - UFPE. Linha de Pesquisa: Percepção e processamento visual da forma em crianças normais e desnutridas, adultos e idosos.

Maria Lúcia de Bustamante Simas é Doutora em Psicologia pela Queen's University, Ontario, Canadá (1985). Prof a Adjunta do Departamento de Psicologia da UFPE. Coordenadora do Laboratótio de Percepção visual — Lab. Vis. Bolsista do CNPq. Linha de Pesquisa: Percepção e Processamento Visual da Forma. 